\title{
Variación del Puntaje Z Peso-edad en lactantes hospitalizados, características de su alimentación y percepción de los padres
}

\section{Variation of Z-score Weight for age in hospitalized infants, feeding characteristics and parental perception}

\author{
Zuny Alejandra Morel ${ }^{1}$, Laura Godoy ${ }^{1}$
}

\section{RESUMEN}

Introducción: La desnutrición en pacientes pediátricos hospitalizados continúa siendo un hallazgo común y una causa frecuente de aumento de la morbimortalidad en niños. Objetivo: Describir la Variación del Puntaje Z del peso-edad en lactantes hospitalizados en sala de internados en el periodo de Septiembre a Diciembre del 2018. Materiales y Métodos: Estudio observacional, descriptivo, transversal, prospectivo con componente analítico. Población: pacientes de 0 a 24 meses hospitalizados en el periodo de septiembre a diciembre del 2018. Variables: edad, sexo, procedencia, tiempo de lactancia materna exclusiva, alimentación actual, alimentación complementaria, comorbilidades, ayuno, peso al ingreso Puntaje $Z$, peso al egreso Puntaje $Z$, diagnóstico nutricional al ingreso, diagnóstico nutricional al egreso, diagnóstico de egreso, percepción de los padres. Análisis de datos: sistema SSPPv21, estadística descriptiva. El protocolo fue aceptado por el comité de ética de la institución con consentimiento informado. Resultados: la mediana de edad fue de 7 meses, predominó el sexo masculino $52 \%$, la mayoría proceden del área central $87 \%$, la mediana del tiempo de lactancia materna exclusiva fue 5 meses, la mediana del inicio de alimentación complementaria fue 6 meses, siendo la mediana de días de internación 4 (mínimo 3 y máximo de 6), la alimentación actual corresponde a LME en $40 \%$,el diagnostico nutricional del ingreso: eutrófico en $65 \%$ y al egreso $66 \%$, el $\mathrm{Pz}$ al ingreso fue $0,29 \pm 0,94 \mathrm{y}$ al egreso fue $0,42 \pm 0,87$. Considerando como punto de corte 5 días de hospitalización, y analizando la variación del Pz (P/E) en relación a los mismos no hubo variación. La percepción de los padres sobre la alimentación de sus hijos durante la hospitalización fue que comen en igual proporción que

\section{ABSTRACT}

Introduction: Malnutrition in hospitalized pediatric patients continues to be a common finding and a frequent cause of increased morbidity and mortality in children. Objective: To describe the variation of the Z-score of the weight for age scale in hospitalized infants from September to December 2018. Materials and Methods: This was an observational, descriptive, cross-sectional, prospective study with an analytical component. Population: patients from 0 to 24 months hospitalized during the time period from September to December 2018. Variables: age, sex, hometown, time of exclusive breastfeeding, current feeding, complementary feeding, comorbidities, fasting, Z-socre weight at admission, Zscore, weight at dicharge, nutritional diagnosis at admission, nutritional diagnosis at discharge, discharge diagnosis, parental perception. Data analysis: SSPPv21 system, descriptive statistics. The protocol was approved by the institution's ethics committee with informed consent. Results: the median age was 7 months, the male sex predominated $(52 \%)$, the majority came from the Central Department $(87 \%)$, the median time of exclusive breastfeeding was 5 months, the median onset of complementary feeding was 6 months, with the median of hospitalization days being 4 (minimum 3 and maximum of 6), current excluside breastfeeing present in $40 \%$, the nutritional diagnosis at admission: eutrophic in $65 \%$ and at discharge $66 \%$, the Z-socre at admission was $0,29 \pm 0.94$ and at discharge was $0.42 \pm 0.87$. Considering 5 days of hospitalization as a cut-off point, and analyzing their relation to the variation of the Z-score (W/A), there was no variation. The perception of the parents about the feeding of their children during hospitalization was that they eat in the same proportion as usual in $74.3 \%$. Conclusion:

\footnotetext{
${ }^{1}$ Ministerio de Salud Pública y Bienestar Social, Hospital Pediátrico "Niños de Acosta Nu". San Lorenzo, Paraguay.

Correspondencia: Laura Godoy. Correo: legodoys@gmail.com

Conflicto de interés: Los autores declaran no poseer conflicto de interés

Recibido: 20/12/2019 Aceptado: 18/02/2020

DOI: https://doi.org/10.31698/ped.47012020005
} 
habitualmente en un 74,3\%. Conclusión: No hubo variación estadísticamente significativa en el Puntaje Z de los pacientes hospitalizados y no se encontró variación del estado nutricional durante hospitalización de los lactantes. La mayoría de los pacientes tuvieron corta estancia hospitalaria y no fueron sometidos a ayuno durante la misma.

Palabras claves: Lactantes, malnutrición, hospitalización, alimentación.

\section{INTRODUCCIÓN}

El término malnutrición abarca dos grupos amplios de afecciones: la desnutrición que comprende el retraso del crecimiento, así como sobrepeso, obesidad y las enfermedades no transmisibles relacionadas con el régimen alimentario ${ }^{(1)}$.

La etiopagenia de la desnutrición en niños es multifactorial: disminución de la ingesta, dolor, aumento de las necesidades metabólicas, y en ocasiones es exacerbada por la enfermedad que motivó hospitalización ${ }^{(2,3,4)}$.

En los niños hospitalizados la desnutrición empeora el estado físico, retarda la cicatrización de los tejidos, produce alteración de los sistemas inmune, cardiológico, respiratorio y digestivo, lo que conlleva una mayor estancia hospitalaria, aumento del número de complicaciones e incremento de los costos de hospitalización. Sin embargo, a pesar del conocimiento de esta realidad, la evaluación nutricional no siempre realiza correctamente en el momento de admisión del paciente en las instituciones hospitalarias ${ }^{(5)}$.

La desnutrición en pacientes pediátricos hospitalizados continúa siendo un hallazgo común y una causa frecuente de aumento de la morbimortalidad en niños ${ }^{(6)}$.

El objetivo del estudio fue analizar la variación del Puntaje Z del peso edad en lactantes hospitalizados al ingreso y al alta. Como objetivo secundario describir el tipo de alimentación (láctea y complementaria), ayuno hospitalario y percepción
There was no statistically significant variation in the Zscore of hospitalized patients and there was no variation in nutritional status during the hospitalization of the infants in this study. Most of the patients had a short hospital stay and were not fasting during that time.

Keywords: Infants, malnutrition, hospitalization, food.

de sus padres respecto sobre los cambios durante la estancia hospitalaria.

\section{MATERIALES Y MÉTODOS}

\section{Diseño y lugar de estudio}

Estudio Observacional, descriptivo, transversal, prospectivo con componente analítico, llevado a cabo en un hospital pediátrico universitario de referencia.

\section{Población y reclutamiento}

Fueron incluidos lactantes de 0 a 24 meses hospitalizados en sala de internados durante el periodo de septiembre a diciembre del 2018, que fueron trasladados desde la sala de urgencias dentro de las $24 \mathrm{hs}$ de admisión y tener el consentimiento informado de los padres. Fueron excluidos aquellos pacientes derivados de otros centros hospitalarios y pacientes reingresados dentro del periodo de estudio. El Muestreo fue no probabilístico por conveniencia de la investigadora. Los lactantes fueron pesados por la misma persona, con el pañal y con balanza pediátrica, mecánica, marca 725 , SECA al ingreso y al alta. Los datos de peso y filiación se introdujeron en el programa Anthros y se utilizó la tabla del puntaje $Z$ peso/edad para niños y niñas de 0 a 5 años de la Organización Mundial de la Salud con los siguientes diagnósticos nutricionales: Eutrófico $(-0,9$ a $+0,9)$, Riesgo de Desnutrición (-1,9 a -1), Desnutrición $(\leq-2)^{(7)}$. Para la variación del Puntaje Z se tomó como punto de corte 5 días, porque es el 
promedio de estancia hospitalaria en el hospital en el que se llevó a cabo el estudio.

Las variables estudiadas fueron: edad, sexo, procedencia, tiempo de lactancia materna exclusiva, alimentación actual, alimentación complementaria, comorbilidades, ayuno, Puntaje $\mathrm{Z}$ peso/edad, al ingreso y al alta, diagnóstico nutricional al ingreso, diagnóstico nutricional al egreso, diagnósticos de egreso, percepción de los padres. En relación al tamaño de la muestra, para conseguir una precisión del 3\% en la estimación de una proporción mediante un intervalo de confianza asintótico normal, con corrección para poblaciones finitas al 95\% bilateral, asumiendo que la proporción esperada es del 11\% y que el tamaño total de la población es de 80, fue necesario incluir 70 lactantes. Los datos fueron analizados con SPSSV21. Las variables cuantitativas de distribución normal se expresaron en medias y desvíos estándar y en medianas con rangos intercuartílicos en caso de distribución no normal Las cualitativas en porcentajes. La asociación de variables cuantitativas se analizó con el test $\mathrm{T}$ Student. La asociación entre variables cualitativas se analizó con el Test Exacto de Fisher. Se respetaron los principios éticos de autonomía, justicia y beneficencia o no maleficencia. El protocolo fue aprobado por el comité de Ética institucional.

\section{RESULTADOS}

Durante el periodo de estudio ingresaron 70 lactantes hospitalizados de 0 a 24 meses. Los datos demográficos se observan en la Tabla 1.

Los lactantes tuvieron una mediana de 4 días de hospitalización con un mínimo de 3 y un máximo de 6 días.

Se analizaron las características de la alimentación de los lactantes (antecedente de lactancia materna y alimentación láctea actual, las mismas se observan en la Tabla 2.

Ningún paciente presentó comorbilidades y ningún paciente estuvo en ayunas durante la hospitalización. $\mathrm{El} \mathrm{Pz} \mathrm{P/E} \mathrm{al} \mathrm{ingreso} \mathrm{fue} \mathrm{de} 0,3 \pm 0,9$ y del peso al egreso fue $0,4 \pm 0,87$. Considerando como punto de corte 5 días de hospitalización y analizando la variación del $\mathrm{Pz}(\mathrm{P} / \mathrm{E})$ en relación a los mismos. Los resultados se describen en la Tabla 3.
Comparando el estado nutricional al ingreso y egreso los resultados se observan en las Tabla 4.

Tabla 1. Datos Demográficos de pacientes internados en el Departamento de Medicina Interna de setiembre a diciembre de 2018. $\mathrm{N}=70$.

\begin{tabular}{llc}
\hline & $\begin{array}{l}\text { Edad }^{\text {(meses) }} \\
\text { Mediana } \\
\text { (P25-P75) }\end{array}$ & 7 \\
& & $\mathbf{( 3 - 1 4 )}$ \\
\hline \multirow{2}{*}{ Sexo } & Masculino & $\mathbf{N}$ (\%) \\
& Femenino & $37(52,9)$ \\
& Central & $33(47,1)$ \\
& Cordillera & $61(87,1)$ \\
& Paraguarí & $3(4,2)$ \\
& Caazapá & $2(2,8)$ \\
& Caaguazú & $1(1,4)$ \\
& Amambay & $1(1,4)$ \\
*Por Departamentos del país. & San Pedro & $1(1,4)$ \\
& & $1(1,4)$ \\
\hline
\end{tabular}

*Por Departamentos del país.

Tabla 2. Características de la Alimentación de los lactantes hospitalizados. $\mathrm{N}=70$.

\begin{tabular}{lll}
\hline Tiempo de lactancia Materna $^{\text {(meses) }}$ & & \\
\hline Mediana & 5 & \\
(P25-P75) & $(2-6)$ & \\
Edad de inicio de Alimentación complementaria ${ }^{\text {(meses) }}$ & & \\
$\quad$ Media (DE) & $6 \pm 2,9$ & \\
Alimentación Láctea actual & $\mathrm{N}$ & $\%$ \\
Leche materna exclusiva & 28 & $(40)$ \\
Leche entera & 27 & $(38,5)$ \\
Leche artificial + Leche materna & 14 & $(20)$ \\
Leche de fórmula & 1 & $(1,4)$ \\
\hline
\end{tabular}

Tabla 3. Score $\mathrm{Z}$ al ingreso y al alta según los días de hospitalización.

\begin{tabular}{lllc}
\hline Días de internación & $<\mathbf{5}$ días & $\geq \mathbf{5}$ días & $\mathbf{P}$ \\
\hline $\begin{array}{l}\text { Pz ingreso } \\
\text { Media DE }\end{array}$ & $0,31 \pm 0.93$ & $0,23 \pm 0,99$ & $0,78^{1}$ \\
$\begin{array}{l}\text { Pz egreso } \\
\text { Media DE }\end{array}$ & $0.45 \pm 0,86$ & $0,36 \pm 0,92$ & $0,82^{1}$ \\
\hline${ }^{1}$ T Student & & &
\end{tabular}

Tabla 4. Estado nutricional al Ingreso y al egreso según percentil $\mathrm{P} / \mathrm{E}$ de pacientes internados en el Departamento Interna de setiembre a diciembre de 2018. $\mathrm{N}=70$.

\begin{tabular}{llllll}
\hline \multicolumn{2}{l}{ Estado Nutricional al Ingreso } & \multicolumn{3}{c}{ Estado Nutricional al egreso } & P \\
\hline & N & \% & N & $\%$ & \\
Eutrófico & 65 & 92.9 & 66 & 94,3 & $0,9^{1}$ \\
R. de Desnutrición & 3 & 4,3 & 2 & 2,9 & \\
Desnutrición & 2 & 2,9 & 2 & 2,9 & \\
Total & 70 & $(100)$ & 70 & 100 \\
\hline test Fisher & & & &
\end{tabular}


Los diagnósticos de egreso se clasificaron según el tipo de patología siendo las más frecuentes las infecciosas 52/70 (74,2\%), seguida de las respiratorias 10/70 (14,2\%), neurológicas 6/70 (8,5\%), hematológicas 1/70 $(1,42)$ y Fallo de Medro 1/70 $(1,42)$.

La mediana de días de internación fue de 4 , con un mínimo de 3 y un máximo de 6 días.

Se evaluó la percepción de los padres en relación a la alimentación durante la hospitalización y los datos se aprecian en la Tabla 5.

Tabla 5. Percepción de los padres sobre la alimentación de sus hijos durante la hospitalización. N=70.

\begin{tabular}{lcc}
\hline Percepción de los padres & N & $\%$ \\
\hline Comen menos & 18 & 25.7 \\
Comen igual & 52 & 74.3 \\
Total & 70 & 100 \\
\hline
\end{tabular}

\section{DISCUSIÓN}

La desnutrición infantil es uno de los problemas principales de Salud Pública de América Latina, los indicadores de crecimiento como el Puntaje Z, pueden aportar datos en pacientes hospitalizados para establecer conductas que eviten la pérdida de peso durante el episodio de enfermedad que enfrentan los niños.

En el presente estudio no se encontró diferencia significativa de variación del Puntaje Z P/E en los lactantes, durante la hospitalización, que puede ser atribuida a las características de la población con mayoría de lactantes que se alimentaban en poco menos de la mitad de los casos con leche materna exclusivamente, además no estuvieron sometidos a ayuno y tuvieron estancia hospitalaria corta. En un estudio realizado por Medina, $S$ y col. en el Hospital Pediátrico Universitario "William Soler" que incluyó a 234 niños de ambos sexos, con edad mayor o igual a 1 año ingresados por más de 24 horas, entre febrero del 2013 y abril del 2014, evaluaron la variación del porcentaje de pérdida de peso, encontraron que la variación promedio en el peso corporal fue de $1,89 \pm 3,01 \%$, en tanto que $51 \%$ no experimentó variación en el peso corporal durante la hospitalización y los niños con estadía hospitalarias prolongadas mostraron pérdidas pronunciada de peso $^{(8)}$.

Un estudio realizado en la Unidad de Gestión Clínica del Niño en el Área de Cuidados Básicos (ACB) del Hospital Padre Hurtado (HPH), de Chile por Acle $\mathrm{M}$ y cols con el objetivo fue analizar los posibles cambios en el estado nutricional durante la hospitalización en pacientes de 29 días a 14 años, no encontraron cambios del estado nutricional según los días de hospitalización y la enfermedad de base. Sin embargo si encontraron una asociación significativa en relación porcentaje de pérdida de peso, los niños desnutridos ganaban peso $0,9 \%$ y los niños con sobrepeso bajan de peso en un $1,3 \%$ ( $p=$ $0,04)$ en la primera semana de hospitalización ${ }^{(9)}$. También se encontraron diferencias con otro estudio realizado por Muñoz y col, en el que incluyeron 206 pacientes hospitalizados en las salas clínicas de la División del Hospital Pediátrico Civil de Guadalajara, México, de los cuales un $40 \%$ eran lactantes, $25 \%$ preescolares, $15 \%$ escolares y $20 \%$ adolescentes. Con las mediciones antropométricas, se estimó la puntuación Z con el programa Antrho y concluyeron que la hospitalización tiene efecto sobre el estado nutricional de los niños, a veces favorable y a otras no. Aquellos pacientes, que tuvieron estancias hospitalarias más largas (más de 10 días), se vieron significativamente afectados al ingreso en los indicadores antropométricos que mostraron un déficit de agudo y estado nutricional crónico en comparación con los que se quedaron menos de 10 días ${ }^{(10)}$. La mediana de días de internación fue de 4 , con un mínimo de 3 y un máximo de 6 días, por lo que se presume no hubo variación en el diagnóstico nutricional de los lactantes hospitalizados, resultados similar a encontrado en el presente estudio, en relación a los días de hospitalización.

Ninguno de los pacientes en este estudio requirió ayuno durante su hospitalización. Un estudio realizado por Acevedo E y col en el Hospital Materno Infantil de la FCM-UNA-Paraguay en el año 2005, incluyó 170 pacientes hospitalizados, y observaron que $12 \%$ de los pacientes presentaron deterioro del estado nutricional durante la internación y que el $80 \%$ fueron sometidos a ayuno con un promedio de 4,4 días $^{(11)}$. Cabe mencionar que en el hospital donde se realizó este trabajo, se 
preconiza el inicio de la alimentación enteral en los pacientes hospitalizados en todos los niveles, buscando un equilibrio entre el riesgo beneficio.

En algunas situaciones la hospitalización, sobre todo si es prolongada, puede tener un impacto en el estado nutricional, ya sea en provocar desnutrición o empeorar una desnutrición previa, esto se puede dar por el estrés que provoca esta situación en el niño lo que le puede llevar a un aumento del catabolismo o al rechazo alimentario ${ }^{(12)}$.

La población de lactantes del presente estudio no presentó comorbilidades y la mayoría eran eutróficos. Muchas patologías preexistentes pueden influir en el estado nutricional por diferentes mecanismos de acuerdo con su naturaleza. En poblaciones con baja comorbilidad hospitalizados, los índices de desnutrición pueden estar por debajo $5 \%$ tal como lo reporta algunos autores ${ }^{(13)}$.

Las patologías más frecuentes de internación fueron las infecciosas en su mayoría, similares al trabajo realizado por Sanabria y col mencionada anteriormente que encontró $66,5 \%$ de infecciones como causa de hospitalización ${ }^{(11)}$.

\section{REFERENCIAS BIBLIOGRÁFICAS}

1. Sullivan PB. Malnutrition in hospitalizedchildren. Arch Dis Child. 2010; 95:489-90.

2. Joosten KF, Zwart H, Hop WC, Hulst JM. National malnutrición screeningdays in hospitalizedchildren in TheNetherlans. Arch Dis Child. 2010; 95:141-5.

3. Moeeni V, Walls T, Day A. Assessment of nutritionalrisk in hospitalizedIranianchildren. Act Pediatr. 2012; 101:e446-51.

4. Cao J, Peng L, Li R, Chen Y, LiX, Mo B. Nutritionalriskscreening and istclinicalsignificance in hospitalizedchildren. Clin Nutr. 2014; 33:432-6.

5. Ramos GR. Desnutrición. In: Loredo AA. Medicina interna pediátrica. México: McGraw-Hill Interamericana; 1996.
La familia es la que más conoce los hábitos alimentarios del niño, que se pueden ver afectados por la hospitalización. Uno de los efectos que se pueden ver sobretodo en lactantes es el aumento del riesgo de pérdida de lactancia materna, efecto observado a $\operatorname{los} 2$ y 6 semanas posterior a la hospitalización ${ }^{(14)}$. Según la percepción de los padres acerca de la alimentación de sus hijos durante ese periodo, no se encontraron diferencias en cuanto a su alimentación habitual.

Es posible que los cambios del estado nutricional se evidencien en hospitalizaciones más prolongadas, en pacientes con comorbilidades, con variaciones del peso para la talla y en pacientes quirúrgicos.

\section{CONCLUSIÓN}

No hubo variación estadísticamente significativa en el Puntaje Z de los lactantes hospitalizados y no se encontró variación del estado nutricional durante la hospitalización. La mayoría eran lactantes con edad mediana de 7 meses eutróficos, sin comorbilidades con corta estadía hospitalaria sin ayuno durante la misma y que se alimentaban en elevado porcentaje con leche materna exclusiva.

6. Von Grebmer K, Ruel MT, Menon P, Nestorova B, Olofinbiyi T, Fritschel H, et al. Índice Global de Hambre. El desafío del hambre: énfasis en la crisis de la subnutrición infantil. Washington, DC: Concern Worldwide; 2010.

7. OMS. Patrones de crecimiento infantil: peso para la edad [Internet]. OMS; 2011 [citado 14 feb 2020]. Disponible en https://www.who.int/childgrowth/standards/peso_para_ edad/es/

8. Medina S, Alfonso L. Variación del peso corporal del niño enfermo durante la hospitalización. Revista Cubana de Alimentación y Nutrición. 2015;25(1):11-27.

9. Acle M, Liberona F. Cambios de peso durante la primera semana de hospitalización en el servicio de pediatría del Hospital Padre Hurtado. [Internet]. 2020 [citado 14 febrero 2020]; Disponible en: http://repositorio.udd.cl/bitstream/ handle/11447/1541/Tesis.pdf?sequence=1 
10. Muñoz-Esparza NC, Vásquez-Garibay EM, RomeroVelarde E, Troyo-Sanromán R. Risk of malnutrition of hospitalized children in a university public hospital. Nutr. Hosp. [Internet]. 2017 [citado 14 Feb 2020]; 34(1): 41-50. Disponible en: https://www.ncbi.nlm.nih.gov/pubmed/ 28244771

11. Acevedo E, Sanabria M, Bellenzier A, Baruja D, Buongermini A, Meza R et al. Evolución del estado nutricional de pacientes pediátricos hospitalizados. Pediatr. (Asunción) [Internet]. 2005 [cited 15 Feb 2020]; 32(2):12-18. Disponible en: http://scielo.iics.una.py/scielo. php?script=sci_abstract\&pid=S1683-98032005000200003 \&lng=en\&nrm=iso\&tlng=es

12. Pérez Moreno J, De la Mata Navazo S, López-Herce Artetaa E, Tolín Hernani M, González Martínez F, González Sánchez MI, et al. Influencia del estado nutricional en la evolución clínica del niño hospitalizado. An Pediatr (Barc). 2019; 91(5):328-335.
13. Moncada Blanco AP, Villanueva Herrera AL. Relación entre estado nutricional y patologías presentes en lactantes y pre-escolares hospitalizados en la E.S.E. Hospital Niño Jesús de Barranquilla enero-marzo 2017 [Internet]. Tesis Pediatría. Barranquilla, Colombia: Universidad Libre Seccional Barranquilla; 2019. [citado 15 Feb 2020] Disponible en http://hdl.handle.net/10901/17728

14. Valderas, J. El niño y su familia: efectos de la hospitalización. Revista de circulación exclusiva para personal médico. 2018; 17(71):3-9. 